\title{
Correlation between RAS Test Results and Prognosis of Metastatic Colorectal Cancer Patients: a Report from Western Iran
}

\author{
Mehrdad Payandeh ${ }^{1}$, Babak Shazad ${ }^{1}$, Masoud Sadeghi ${ }^{1 *}$, Maryam Shahbazi² $^{2}$
}

\begin{abstract}
In the patients with metastatic colorectal cancer (mCRC), RAS testing is the first step to identify those that could benefit from anti-EGFR therapy. This study examined associations between KRAS mutations and clinicopathological and survival data in Iranian patients with mCRC. Between 2008 to2015 in a retrospective study, 83 cases of $\mathbf{m C R C}$ were referred to the Clinic of Medical Oncology. The mean follow-up was 45 months that there were 27 deaths. The 3 patients that did not complete follow-up were censored from the study. KRAS and NRAS were analyzed using allele-specific PCR primers and pyrosequencing in exons 2,3 and 4. Multivariate survival analysis using Cox's regression model was used for affecting of variables on overall survival (OS). The mean age at diagnosis for patients was 57.7 (range, 18 to 80 years) and $61.4 \%$ were male. There was no significant different between prognostic factors and KRAS mutation with wild-type. Also, There was no significant different between KRAS mutation and KRAS wild-type for survival, but there was a significant different between KRAS 12 and 13 mutations for survival (HR 0.13, 95\% CI 0.03-0.66, P=0.01). In conclusion, the prevalence of KRAS mutations in CRC patients was below $50 \%$ but higher than in other studies in Iran. As in many studies, patients with KRAS 12 mutations had better OS thn those with KRAS 13 mutation. In addition to KRAS testing, other biomarkers are needed to determine the best treatment for patients with mCRC.
\end{abstract}

Keywords: KRAS - NRAS - metastatic colorectal cancer - survival - western Iran

Asian Pac J Cancer Prev, 17 (4), 1729-1732

\section{Introduction}

The development of colorectal cancer (CRC) is a multistep process that occurs because of the accumulation of several genetic alterations, including chromosomal abnormalities, gene mutations, and epigenetic modifications involving several genes that regulate proliferation, differentiation, apoptosis, and angiogenesis (Russo et al., 2009). In the patients of metastatic CRC (mCRC), RAS testing is the first step to identify those patients that could benefit from anti-EGFR therapy (Payandeh et al., 2016). Mutation of the KRAS gene plays an important role in colorectal tumorigenesis (Li et al., 2012). KRAS mutations in CRC have been stated to be 20-50\% (Shen et al., 2011). NRAS/BRAF mutation probably is effective in the treatment of CRC patients with KRAS wild-type and in patients with KRAS wild-type should be specified NRAS/BRAF testing to determine which patients will benefit from anti-EGFR therapy (Payandeh et al., 2015a). There are very few studies about KRAS mutations in CRC from developing countries such as Iran (Omidifar et al., 2015). Cetuximab plus FOLFOX (fluorouracil + leucovorin + oxaliplatin) is more effective in achieving a greater response rate and lower risk of disease progression in KRAS wild-type than KRAS mutation CRC (Bokemeyer et al., 2009). Totally, in retrospective analyzes have been shown that patients with KRAS wild-type had better OS compared to patients harboring KRAS mutation (Lievre et al., 2006).

This study examined associations between KRAS mutations and clinicopathological and survival data in Iranian patients with $\mathrm{mCRC}$.

\section{Materials and Methods}

\section{Patients}

Between of 2008 to 2015 in a retrospective study, 83 patients with mCRC referred to Clinic of Medical Oncology, Kermanshah city, Iran. Age, sex, type of pathology, tumor size, differentiation, site of tumor and metastasis, KRAS/NRAS testing, lymph node metastasis and survival were checked. The mean follow-up was 45 months that there were 27 deaths and also 3 patients did not complete follow-up that censored from study. OS was 
Mehrdad Payandeh et al

defined from the date of diagnosis until death from any cause or the date of the last follow-up.

\section{Analysis of KRAS/NRAS}

Specimen was FFPE tissue block including $80 \%$ tumor. DNA extracted by FFPE QIAGEN kit and KRAS and NRAS were analyzed using allele-specific PCR primers and pyrosequencing. The results have been double checked by high resolution melting analysis. Detection limit of this assay is five copies of mutations in whole genome. Checked mutations for KRAS and NRAS were:

KRAS mutations: Exon2: Codons 12 and 13; Exon3: Codons 59, 176, 181, 182 and 183; Exon4: Codons 146 and 117.

NRAS mutations: Exon2: Codons 12 and 13; Exon3: Codons 59 and 61; Exon4: Codons 146 and 117.

Misinterpretation of results may occur if the information provided is inaccurate or incomplete. Every molecular test has a $0.5-1 \%$ error rate. This is due to rare molecular events and factors related to the preparation and analysis of samples.

\section{Statistical analysis}

Analysis of data was done by IBM SPSS version 19 (SPSS Inc., Chicago, IL, USA) software and survival diagram was plotted by GraphPad Prism version 5.04 software. Multivariate survival analysis using Cox's regression model was used for affecting of variables on OS. P-value $<0.05$ was considered statistically.

\section{Results}

The mean age at diagnosis for patients was $57.7 \pm 13$ years (range, 18 to 80 years) that 51 patients $(61.4 \%$ ) were male (Table 1). 50 patients $(60.2 \%), 27(32.5 \%)$ and $6(7.3 \%)$ were well, moderate and poorly differentiated, respectively. Out of 83 patients, type of pathology in 51 patients $(61.4 \%)$ were adenocarcinoma, site of tumor in 32 patients $(38.6 \%$ ) was rectum, KRAS testing for 46 patients (55.4\%) was wild-type, NRAS testing for all patients was wild-type, site of metastasis for 55 patients $(66.3 \%)$ was liver and lymph node metastasis for $55(71.4 \%$ ) was positive.

Table 2 shows the prevalence of KRAS mutations in exons 2, 3 and 4. G12D codon was the most mutation for codon 12 and G13D is only mutation in codon 13 .

The correlation of prognostic factors between KRAS

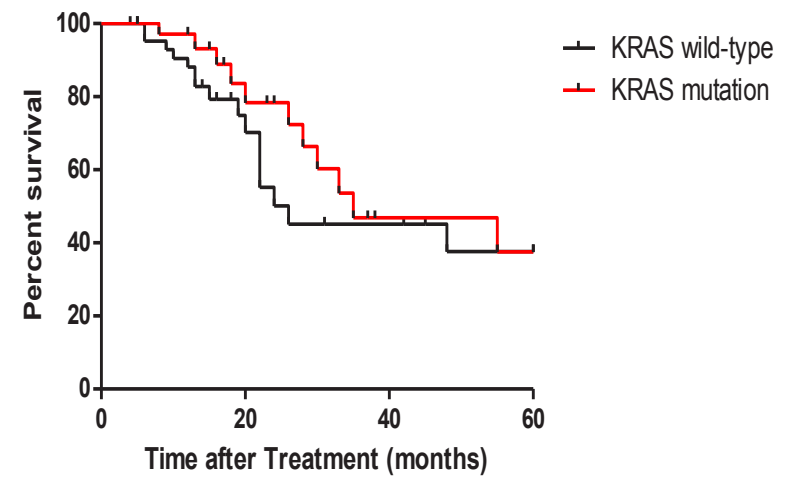

Figure 1. 5-Year Overall Survival in Patients with Metastatic Colorectal Cancer Based on KRAS Testing
Table 1. Baseline Variables in Patients with Metastatic Colorectal Cancer $(n=83)$

\begin{tabular}{|c|c|c|c|}
\hline Variables & $\mathrm{n}(\%)$ & Mean \pm SD & Range \\
\hline Age, years & & $57.7 \pm 13.0$ & $28-80$ \\
\hline \multicolumn{4}{|l|}{ Sex } \\
\hline Male & $51(61.4)$ & & \\
\hline Female & $32(38.6)$ & & \\
\hline \multicolumn{4}{|l|}{ Differentiation } \\
\hline Poorly differentiated & $6(7.3)$ & & \\
\hline Moderate differentiated & $27(32.5)$ & & \\
\hline Well differentiated & $50(60.2)$ & & \\
\hline \multicolumn{4}{|l|}{ Tumor site } \\
\hline Rectum & $32(38.6)$ & & \\
\hline Sigmoid & $21(25.3)$ & & \\
\hline Rectosigmoid & $9(10.8)$ & & \\
\hline Descending Colon & $3(3.6)$ & & \\
\hline Transverse Colon & $6(7.2)$ & & \\
\hline Ascending Colon & $5(6.1)$ & & \\
\hline Cecum & $7(8.4)$ & & \\
\hline \multicolumn{4}{|l|}{ Type of pathology } \\
\hline Adenocarcinoma & $51(61.4)$ & & \\
\hline Mucinous & $32(38.6)$ & & \\
\hline Tumor size, $\mathrm{cm}$ & & $5.5 \pm 2.2$ & $1.5-12$ \\
\hline \multicolumn{4}{|l|}{ KRAS } \\
\hline Wild-type & $46(55.4)$ & & \\
\hline Mutation & $37(44.6)$ & & \\
\hline \multicolumn{4}{|l|}{ NRAS } \\
\hline Wild-type & $83(100)$ & & \\
\hline Mutation & 0 & & \\
\hline \multicolumn{4}{|l|}{ Site of metastasis } \\
\hline Liver & $55(66.3)$ & & \\
\hline Lung & $6(7.2)$ & & \\
\hline Small intestinal & $5(6.1)$ & & \\
\hline Bone & $4(4.8)$ & & \\
\hline Others & $13(15.6)$ & & \\
\hline \multicolumn{4}{|l|}{ Lymph node metastasis } \\
\hline Yes & $55(71.4)$ & & \\
\hline No & $28(28.6)$ & & \\
\hline
\end{tabular}

Table 2. Prevalence of KRAS Codon Mutations $(n=37)$

\begin{tabular}{lc}
\hline Mutation & $\mathrm{n}(\%)$ \\
\hline $\mathrm{G} 12 \mathrm{D}(\mathrm{c} .35 \mathrm{G}>\mathrm{A})$ & $12(32.5)$ \\
$\mathrm{G} 12 \mathrm{~V}(\mathrm{c} .35 \mathrm{G}>\mathrm{T})$ & $8(21.6)$ \\
$\mathrm{G} 12 \mathrm{~S}(\mathrm{c} .34 \mathrm{G}>\mathrm{A})$ & $4(10.8)$ \\
$\mathrm{G} 12 \mathrm{C}(\mathrm{c} .34 \mathrm{G}>\mathrm{T})$ & $3(8.1)$ \\
$\mathrm{G} 12 \mathrm{~A}(\mathrm{c} .35 \mathrm{G}>\mathrm{C})$ & $2(5.4)$ \\
$\mathrm{G} 12 \mathrm{R}(\mathrm{c} .34 \mathrm{G}>\mathrm{C})$ & $1(2.7)$ \\
$\mathrm{G} 13 \mathrm{D}(\mathrm{c} .38 \mathrm{G}>\mathrm{A})$ & $7(18.9)$ \\
\hline
\end{tabular}

$\overline{\text { G: Glycine (Gly); A: Alanine (Ala); D: Aspartic acid (Asp); S: Serine }}$ (Ser); C: Cysteine (Cys); R: Arginine (Arg); V: Valine

wild-type and KRAS mutation has been shown in Table 3. There was no significant different between these factors and KRAS mutation with wild-type.

Median 5-year survival for KRAS mutation patients was 35 months and for KRAS wild type was 26 months (Figure 1).Also, the survival rate for KRAS mutation and KRAS wild type was $69 \%$ and $64 \%$, respectively. There was no significant different between KRAS mutation and KRAS wild-type for survival (HR $1.43,95 \%$ CI 0.663.08, $\mathrm{P}=0.35$ ).

Median 5-year survival in patients with KRAS codon 12 mutations was 55 and codon 13 mutation was 26 months (Figure 2). Also, the survival rate for KRAS 
RAS Mutations and Prognosis of Metastatic Colorectal Cancer Patients: a Report from Western Iran

Table 3. The Correlation of Prognostic Factors between KRAS wild-type and KRAS Mutation

\begin{tabular}{|c|c|c|c|}
\hline \multirow[t]{2}{*}{ Variable } & $\begin{array}{c}\text { KRAS } \\
\text { wild-type }\end{array}$ & $\begin{array}{l}\text { KRAS } \\
\text { mutation }\end{array}$ & P-value \\
\hline & $\mathrm{N}=46$ & $\mathrm{~N}=37$ & \\
\hline Mean age, years & 56.6 & 58.9 & 0.427 \\
\hline Sex & & & 0.307 \\
\hline Male & $30(65.2)$ & $21(56.8)$ & \\
\hline Female & $16(34.8)$ & $16(43.2)$ & \\
\hline Differentiation & & & 0.631 \\
\hline Poorly differentiated & $4(8.7)$ & $2(5.4)$ & \\
\hline Moderate differentiated & $15(32.6)$ & $12(32.4)$ & \\
\hline Well differentiated & 27(58.7) & $23(62.2)$ & \\
\hline Tumor site & & & 0.53 \\
\hline Rectum & 34(73.9) & $28(75.7)$ & \\
\hline Colon & $12(26.1)$ & $9(24.3)$ & \\
\hline Type of pathology & & & 0.365 \\
\hline Adenocarcinoma & $27(58.7)$ & $24(64.9)$ & \\
\hline Mucinous & $19(41.3)$ & $13(35.1)$ & \\
\hline Mean size of tumor, $\mathrm{cm}$ & 5.6 & 5.5 & 0.856 \\
\hline Lymph node metastasis & & & 0.689 \\
\hline Yes & $30(65.2)$ & $25(67.6)$ & \\
\hline No & $16(34.8)$ & $12(32.4)$ & \\
\hline
\end{tabular}

Table 4. Multivariate Survival Analysis Using Cox's Regression Model for Affecting of Variables on Overall Survival

\begin{tabular}{lccc}
\hline \multicolumn{1}{c}{ Variables } & P-value & HR* & 95\% CI \\
\hline Age(years), $\geq 55 \mathrm{v}<55$ & 0.28 & 0.65 & $0.29-1.43$ \\
$\begin{array}{l}\text { Sex, male v female } \\
\begin{array}{l}\text { Differentiation, well v } \\
\text { moderate or poorly }\end{array}\end{array}$ & 0.01 & 0.32 & $0.13-0.79$ \\
$\begin{array}{l}\text { Tumor site, rectum v colon } \\
\text { Type of pathology, }\end{array}$ & 0.42 & 0.75 & $0.38-1.49$ \\
$\begin{array}{l}\text { adenocarcinoma v mucinous } \\
\begin{array}{l}\text { Lymph node metastasis, yes } \\
\text { v no }\end{array}\end{array}$ & 0.39 & 1.46 & $0.54-4.48$ \\
\hline
\end{tabular}

*HRs are presented as the risk of the right-side category (ie, right side of $\mathrm{v}$ in Parameter column) to the left-side category (ie, left side of $\mathrm{v}$ in Parameter column). Abbreviation: HR, hazard ratio

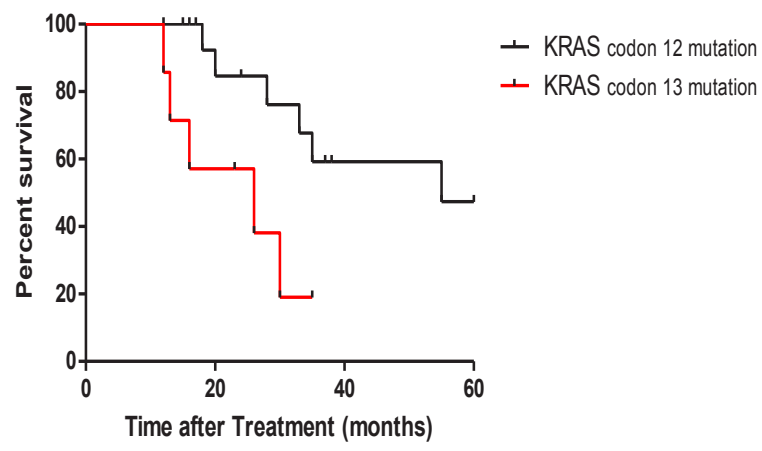

Figure 2. 5-Year Overall Survival in Patients with Metastatic Colorectal Cancer Based on KRAS Codon Mutations

codon 12 mutations and codon 13 mutations was $79.3 \%$ and $28.5 \%$, respectively. There was a significant different between KRAS 12 mutations and KRAS 13 mutation for survival (HR 0.13, 95\% CI 0.03-0.66, $\mathrm{P}=0.01$ ).

A Cox proportional hazard regression analysis was used to assess the effects of various parameters on the primary analysis. Male sex is an unfavorable predictor for OS of KRAS wild-type compared to KRAS mutation patients (Table 4).

\section{Discussion}

Approximately $30 \%$ to $50 \%$ of colorectal tumors are known to have a mutated KRAS gene, indicating that up to $50 \%$ of patients with CRC might respond to anti-epidermal growth factor receptor (EGFR) antibody therapy. However, $40 \%$ to $60 \%$ of patients with wild-type KRAS tumors do not respond to such therapy (Wilson et al., 2010). Based on 3 studies in Iran on CRC patients, the prevalence of KRAS mutation was between $12.5 \%$ in Tehran to $37.4 \%$ in this city (Central Iran) and also based on the studies in Asian other countries, between $23 \%$ in Thailand to $50 \%$ in Japan. In European countries, KRAS mutation was $30 \%$ in Italy to $49.3 \%$ in the UK, the USA (31.6\%), Australia (28\%), and African countries (23-28\%). In this study (Western Iran), the prevalence was $44.6 \%$ (Omidifar et al., 2015). Therefore, the results show that percentage of KRAS mutations in CRC patients is below of $50 \%$ and in our study was higher than other studies in Iran. In more studies, $12 \mathrm{G}>\mathrm{A}$ was the most common substitution for KRAS Codon 12 (Omidifar et al., 2015) similar to our study. Therefore, ethnicity (Al-Kuraya, 2009; Sameer et al., 2009) and geography (Neumann et al., 2009; Brink et al., 2005) probably can effect on the prevalence of KRAS mutation.

Data from a large Japanese population of patients with advanced and recurrent CRC showed that the prevalence KRAS mutation is approximately $35 \%$ that $25 \%$ patients had mutations at codon 12 and $10 \%$ patients had mutations at codon 13 (Yokota et al., 2010). Approximately 40\% of CRCs harbor KRAS mutations, and $90 \%$ of those mutations occur in codons 12 and 13 (Roock et al., 2011). Of $44.6 \%$ KRAS mutation in this study, KRAS codon 12 mutation $(36.2 \%)$ was more than codon $13(8.4 \%)$. In one study (Li et al., 2012), patients with KRAS wildtype had the significantly higher median survival times than patients with KRAS mutations (35.05 vs. 25.72 months). Another study (Margonis et al., 2015), reported that median and 5-year survival rate among patients with KRAS mutation was 32.4 months and $32.7 \%$, respectively, vs. 58.5 months and $46.9 \%$, respectively, for patients with KRAS wild-type $(\mathrm{P}<0.05)$. A total of 99 patients with stage I-IV CRC, the cumulative 5-year survival rates for patients with KRAS wild-type, KRAS 12 and KRAS 13 mutations were $81.4,61.4$ and $42.0 \%$, respectively $(\mathrm{P}<0.05)$ (Inoue et al., 2012). Omidifar et al. (2015) showed that KRAS 13 mutation is associated with poor prognostic outcomes, including reduced survival rate, less stable cancer and disease relapse compared with codon 12 mutations. In the study of Margonis et al. (2015), patients with KRAS 12 mutations had worse OS vs. those with KRAS wild-type, whereas a KRAS codon 13 mutation was not associated with prognosis. Compared with KRAS codon 12 mutations, codon 13-mutated mCRC presents as a more aggressive disease frequently associated with local and distant metastases at first diagnosis (Modest 
et al., 2011). In another research ( $\mathrm{Li}$ et al., 2012), has been reported that KRAS mutations at codons 12 or 13 did not have the significantly different median survival times. In our study, median and 5-year survival rate for KRAS mutation and KRAS wild-type were 35 months and $69 \%$, respectively, 26 months and $64 \%$, respectively. Also, patients with KRAS 12 mutations had better OS vs. those with KRAS 13 mutation that that agrees with our study. Median and survival rate for KRAS 12 mutations and codon 13 mutation was 55 months and $79.3 \%$, respectively, vs. 26 months and $28.5 \%$, respectively. OS in KRAS mutation/NRAS wild-type patients was higher than KRAS/NRAS wild-type ( $>>0.05)$, contrasting with the results of other studies (Tan and Du,2012; Payandeh et al., 2015b). Multivariate survival analysis showed that male sex was an unfavorable predictor for OS. Allegra et al. (2016) reported that all patients with mCRC who are candidates for anti-EGFR therapy should have their tumor tested for mutations in both KRAS and NRAS. In addition to RAS testing, other biomarkers are needed to determine the best treatment for patients with $\mathrm{mCRC}$, because the efficacy of anti-EGFR therapy, even in the RAS wildtype population, is modest. KRAS gene mutations were significantly associated with poor tumor differentiation and liver metastasis (Li et al., 2012). In the study of Liu et al. (2011), clinicopathologic features (age, sex, and tumor site, depth, size, grade, and metastasis) were not different between KRAS mutation and wild-type CRCs. In this study, there was no significant correlation between prognostic factors (age, sex, tumor size, differentiation, lymph node metastasis, type of pathology and tumor site) with KRAS mutation and wild-type. Therefore, the efficacy of anti-EGFR therapy is no suitable in all KRAS wild-type patients and we should be looking for other effective factors.

In conclusions, the prevalence of KRAS mutations in CRC patients is below of $50 \%$ and in our study was higher than other studies in Iran. In a lot of studies, patients with KRAS 12 mutations had better OS vs. those with KRAS 13 mutation. In addition to KRAS/NRAS testing, other biomarkers are needed to determine the best treatment for patients with mCRC.

\section{Acknowledgements}

Merck Serono Company was as the sponsor for this research and the authors thank for its supporting.

\section{References}

Allegra CJ, Rumble RB, Hamilton SR, et al (2016). Extended ras gene mutation testing in metastatic colorectal carcinoma to predict response to anti-epidermal growth factor receptor monoclonal antibody therapy: american society of clinical oncology provisional clinical opinion update 2015. J Clin Oncol, 34, 179-85.

Al-Kuraya KS (2009). KRAS and TP53 mutations in colorectal carcinoma. Saudi J Gastroenterol, 15, 217-9.

Brink M, Weijenberg MP, de Goeij AF, et al (2005). Dietary folate intake and k-ras mutations in sporadic colon and rectal cancer in The Netherlands Cohort Study. Int J Cancer, 114, 824-30.
Bokemeyer C, Bondarenko I, Makhson A, et al (2009). Fluorouracil, leucovorin, and oxaliplatin with and without cetuximab in the first-line treatment of metastatic colorectal cancer. J Clin Oncol, 27, 663-71.

Inoue Y, Saigusa S, Iwata T, et al (2012). The prognostic value of KRAS mutations in patients with colorectal cancer. Oncol Rep, 28, 1579-84.

Lievre A, Bachet J, Le Corre D, et al (2006). KRAS mutation status is predictive of response to cetuximab therapy in colorectal cancer. Cancer Res, 66, 3992-5.

Liu X, Jakubowski M, Hunt JL (2011). KRAS gene mutation in colorectal cancer is correlated with increased proliferation and spontaneous apoptosis. Am J Clin Pathol, 135, 245-52.

Li Z, Chen Y, Wang D, et al (2012). Detection of KRAS mutations and their associations with clinicopathological features and survival in Chinese colorectal cancer patients. $J$ Int Med Res, 40, 1589-98.

Margonis GA, Kim Y, Spolverato G, et al (2015). Association between specific mutations in KRAS codon 12 and colorectal liver metastasis. JAMA Surg, 150, 722-9.

Modest DP, Stintzing S, Laubender RP, et al (2011). Clinical characterization of patients with metastatic colorectal cancer depending on the KRAS status. Anticancer Drugs, 22, 913-8.

Neumann J, Zeindl-Eberhart E, Kirchner T, et al (2009). Frequency and type of KRAS mutations in routine diagnostic analysis of metastatic colorectal cancer. Pathol Res Pract, 205, 858-62.

Omidifar N Md, Geramizadeh B Md, Mirzai M Ms (2015). K-ras mutation in colorectal cancer, a report from southern Iran. Iran J Med Sci, 40, 454-60.

Payandeh M, Sadeghi E, Sadeghi M, et al (2015a). Chronic myeloid leukemia in patient with local recurrence colon cancer: a case report. Int J Cancer Research, 11, 197-200.

Payandeh M, Sadeghi M, Sadeghi E, et al (2015b). Analysis of KRAS, BRAF and NRAS in Patients with Colorectal Cancer: the First Report of Western Iran. Am J Cancer Prevent, 3, 19-22.

Payandeh M, Sadeghi M, Sadeghi E (2016). The report of KRAS mutation and NRAS wild-type in a patient with thyroid metastasis from colon cancer: a rare case report. Iran $J$ Pathol, 11, 71-5.

Russo A, Rizzo S, Bronte G, et al (2009). The long and winding road to useful predictive factors for anti-EGFR therapy in metastatic colorectal carcinoma: the KRAS/BRAF pathway. Oncol, 77, 57-68.

Roock WD, Vriendt VD, Normanno N, et al (2011). KRAS, BRAF, PIK3CA, and PTEN mutations: implications for targeted therapies in metastatic colorectal cancer. Lancet Oncol, 12, 594-603.

Sameer AS, Chowdhri NA, Abdullah S, et al (2009). Mutation pattern of K-ras gene in colorectal cancer patients of Kashmir: a report. Indian J Cancer, 46, 219-25.

Shen H, Yuan Y, Hu HG, et al (2011). Clinical significance of $\mathrm{K}$-ras and BRAF mutations in Chinese colorectal cancer patients. World J Gastroenterol, 17, 809-16.

Tan C, Du X (2012). KRAS mutation testing in metastatic colorectal cancer. World J Gastroenterol, 18, 5171-80.

Wilson PM, Labonte MJ, Lenz HJ (2010). Molecular markers in the treatment of metastatic colorectal cancer. Cancer $J$, 16, 262-72.

Yokota T, Shibata N, Ura T, et al (2010). Cycleave polymerase chain reaction method is practically applicable for V-Ki-ras2 Kirsten rat sarcoma viral oncogene homolog (KRAS)/Vraf murine sarcoma viral oncogene homolog B1 (BRAF) genotyping in colorectal cancer. Transl Res, 156, 98-105. 